D.O.I.:10.3895/S1808-04482006000200012

\title{
SEIS SIGMA: MELHORIA DA QUALIDADE ATRAVÉS DA REDUÇÃO DA VARIABILIDADE
}

\section{SIX SIGMA: IMPROVEMENT OF THE QUALITY THROUGH THE REDUCTION OF THE VARIABILITY}

\author{
Sidarta Ruthes ${ }^{1}$; Paulo Sérgio Ceretta ${ }^{2}$; Igor Bernardi Sonza ${ }^{3}$ \\ ${ }^{1}$ Universidade Tecnológica Federal do Paraná - UTFPR - Curitiba - Brasil \\ sidarta@ppgte.cefetpr.br \\ ${ }^{2}$ Universidade Federal de Santa Maria - UFSM - Santa Maria - Brasil \\ ceretta@smail.ufsm.br \\ ${ }^{3}$ Universidade Federal de Santa Maria - UFSM - Santa Maria - Brasil \\ ibs182@terra.com.br
}

\begin{abstract}
Resumo
Dentro do contexto da busca por produtos perfeitos, encontram-se várias estratégias que visam aumentar a vantagem competitiva das organizações, dentre elas existe o Seis Sigma. Este artigo visa comparar os beneficios operacionais que o programa Seis Sigma pode proporcionar no atual processo de produção de refrigerante pet $2000 \mathrm{ml}$ da empresa X. No desenvolvimento deste artigo foi utilizada uma pesquisa exploratória, a fim de investigar e proporcionar uma visão geral, de tipo aproximativo, acerca do programa Seis Sigma em relação ao processo de fabricação do refrigerante pet $2000 \mathrm{ml}$. Neste estudo, ficou evidente que em termos operacionais o programa Seis Sigma pode trazer, se bem aplicado, grandes beneficios para o processo de produção do refrigerante pet 2 litros, com redução do número de defeitos e, conseqüentemente, um aumento da uniformidade dos produtos.
\end{abstract}

Palavras-chave: seis sigma; controle de qualidade; gerenciamento de processos.

\section{Introdução}

Atualmente os mercados estão cada vez mais competitivos e a fidelidade dos clientes cada vez mais disputada. As empresas que buscam o crescimento e almejam o mercado mundial, devem comercializar produtos e serviços de forma a "encantar" seus clientes. Para surpreender os consumidores, com produtos interessantes, preços atrativos e de excelente qualidade, são necessários várias horas de muito empenho e dedicação na elaboração de projetos consistentes. A busca incessante por produtos e serviços perfeitos leva muitas pessoas a pesquisar e implantar várias técnicas, ferramentas e estratégias. Dentro do contexto da busca por produtos perfeitos, 
encontram-se várias estratégias que visam aumentar a vantagem competitiva das organizações, entre elas, o Seis Sigma.

De acordo com um dossiê publicado pela Revista HSM Management (2003), na década de 80, o presidente da Motorola, Robert Garvin, juntamente com sua equipe, criaram o programa de qualidade batizado com o nome 6-sigma. O engenheiro da Motorola Bill Smith fez parte da equipe de Garvin e sua participação foi fundamental na percepção de que o controle da variação na produção poderia alcançar um resultado final de 3,4 defeitos por milhão de oportunidades, ou seja, a oportunidade de defeitos no nível 6-sigma.

Os resultados alcançados com o programa Seis Sigma estimularam vários estudos e pesquisas sobre o tema. Para McCarthy e Stauffer (2001), o seis sigma é a mais poderosa força para corporações que precisam mudar suas operações e seus processos de desenvolvimento de produtos e serviços. Empresas como a General Eletric, Motorola e Ford investiram, aproximadamente, 100 milhões de dólares em projetos Seis sigma, com expectativas de receber bilhões de dólares em contrapartida. A ênfase do Seis sigma está nos resultados financeiros e na possibilidade de eliminação de produtos e processos defeituosos. Segundo McCarthy e Stauffer (2001), acabou-se os dias da qualidade a qualquer custo. Atualmente, os programas de qualidade precisam trazer resultados mensuráveis.

Este artigo tem como objetivo comparar duas etapas do processo de fabricação do refrigerante pet 2 litros com a produção em um nível de variação de processo Seis Sigma, identificando e explicando alguns benefícios que o programa Seis Sigma pode proporcionar para a empresa X. Inicialmente será apresentada uma revisão literária, por meio de referências de publicações a respeito de qualidade e da "filosofia" Seis Sigma, após será explicitada a metodologia aplicada neste estudo, com a caracterização do trabalho e, por fim, a análise dos dados empíricos e os resultados obtidos com a projeção do Seis Sigma.

\section{Qualidade Rumo à Perfeição}

A qualidade não é temporária e nem é obra do acaso. De acordo com Bateman e Snell (1998), pouco se pode fazer, em uma base temporária, para melhorar a qualidade. Alguns gerentes têm tentado implementar um programa da qualidade temporário ou sob forma de campanha, geralmente incluindo reuniões com empregados, seminários, posters, slogans, emblemas e artigos em boletins de informações dos empregados. A qualidade é produto de uma cultura organizacional que orienta a melhoria contínua e a preocupação em produzir mercadorias e serviços de alta performance. Se os empregados acreditam que o programa da gerência é passageiro, eles o tratarão como tal. Depois de instalado um programa passageiro, a qualidade do produto pode até alcançar 
uma certa melhoria, mas ele não permanecerá nesse novo nível e certamente essa melhoria não irá além daquele ponto. Os programas improvisados são, na melhor das hipóteses, transitórios.

A qualidade nas organizações vem evoluindo em todos os aspectos. A abordagem de Garvin (1992) define qualidade considerando a visão de quem a percebe, por exemplo: (i) transcendente, que define qualidade como excelência inata; (ii) produto, quando descreve a qualidade como algo preciso e mensurável; (iii) usuário, quando a qualidade está diante dos olhos de quem observa; (iv) produção, quando a conformidade às especificações é qualidade; e, (v) valor, quando a qualidade está diretamente relacionada aos custos e preços. Davis, Aquilano e Chase (2001) divide a qualidade em duas categorias: (i) qualidade do produto e, (ii) qualidade do processo. O nível de qualidade na elaboração de um produto irá variar com relação ao mercado específico que ele almeja atender e, no sentido de processo, qualidade é produzir produtos livres de erros.

Lobos (1991), na tentativa de definir "zero defeito", ou como prefere "zero erro", descreve a importância da qualidade para empresas que possuem produtos complexos, com agregação de várias peças e componentes até formar o produto final. $\mathrm{O}$ autor lembra o episódio da Motorola, quando antes da implantação do Seis Sigma, na produção de uma central telefônica, que possuía 1.200 componentes e uma tolerância de 2.700 componentes defeituosos em 1 milhão, o que eqüivale a 99,7\% de produtos perfeitos, a combinação de componentes defeituosos e nãodefeituosos se multiplicavam de tal forma que, no final das contas, num lote de 1.000 centrais telefônicas tinha-se 40 que acabavam rejeitadas. Lobos (1991) salienta que a disposição ao "zero erro" não é um compromisso com a utopia, mas com a perfeição possível, representa uma nova forma de raciocínio segundo a qual os ganhos de produtividade ou de qualidade só podem ser de natureza infinitesimal.

Existem algumas estratégias para se alcançar uma produção com "zero erro". Pande, Neuman e Cavanagh (2001) afirmam que há três estratégias Seis Sigma. As estratégias são: (i) estratégia de melhoria de processo; (ii) estratégia de projeto/reprojeto de processo; e, (iii) estratégia de gerenciamento de processo. A melhoria de processo refere-se à estratégia de desenvolver soluções com a finalidade de eliminar as causas-raiz dos problemas de desempenho de uma empresa, sem, no entanto, interferir na estrutura básica do processo. Na estratégia projeto/reprojeto de processo, o objetivo é substituir uma parte ou todo o processo por um novo. Já na estratégia de gerenciamento de processo, as exigências do cliente são claras e regularmente atualizadas, os processos são documentados e gerenciados com medições em todas as suas etapas. Nesta última estratégia, os gestores também usam as medições e o conhecimento do processo para avaliar os seus desempenhos.

O Seis Sigma é um sistema que liga idéias, tendências e ferramentas desconexas nos negócios, onde o foco no cliente torna-se a prioridade principal. As melhorias Seis Sigma são 
definidas pelo seu impacto sobre a satisfação e valores dos clientes. Existem muitas decisões de negócios que se baseiam em opiniões e suposições. A disciplina Seis Sigma começa esclarecendo que medidas são a chave para avaliar o desempenho dos negócios; depois, aplica-se dados e análises de modo a se construir um entendimento das variáveis-chaves e a otimizar resultados. A Figura 1 resume alguns métodos importantes do programa Seis Sigma. (PANDE, NEUMAN e CAVANAGH, 2001).

Figura 1 - Métodos e Ferramentas Essenciais do Programa Seis Sigma

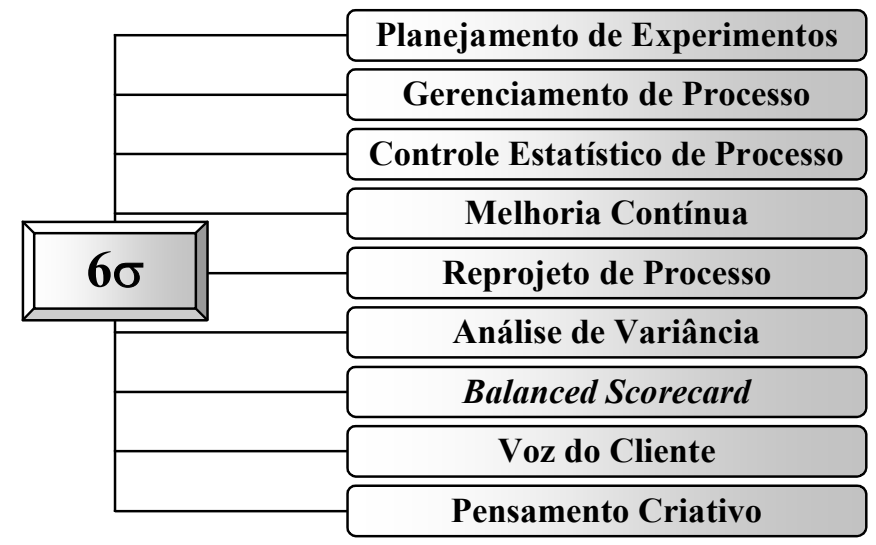

Fonte: Adaptado de PANDE; NEUMAN; CAVANAGH. (2001, p. 15).

\section{A Estatística no Controle da Qualidade}

Conforme Hutchins (1994), o controle estatístico da qualidade (SQC) originou-se na década de 80, no setor comercial. Com base no SQC, surgiu, anos mais tarde, a filosofia Seis Sigma. A filosofia do programa Seis Sigma enfatiza um controle estatístico da qualidade que tenta definir os padrões da excelência operacional. Esta filosofia esforça-se para alcançar operações com não mais de 3,4 defeitos por um milhão. O Seis Sigma é orientado para prevenção, tornando-se um dos principais impulsos para a medição da melhoria contínua e para a fixação de níveis referenciais competitivos. Breyfogle (2003) afirma que se os dados fossem concentrados dentro dos limites de especificações e tivesse vários desvios padrões à taxa de ppm (parts per million) representaria o número de partes por milhão que estariam fora dos limites, conforme a Tabela 1.

Campos (2002) assegura que o Controle de Qualidade Total (TQC) e a abordagem Seis Sigma apoiam-se em ferramentas comuns, mas a gestão das duas estratégias é bastante diferentes. $\mathrm{O}$ TQC está fundamentado no aprimoramento contínuo, sendo considerado uma jornada em que nunca se atinge o alvo, pois ele está sempre à frente, ou seja, quando se atinge um certo patamar, busca-se outro mais adiante. Já o programa Seis Sigma procura buscar a perfeição na resolução de projetos, com alvos bem definidos, onde se sabe quando os objetivos foram alcançados. Campos (2002) salienta, também, que o Seis Sigma incorpora a visão do negócio na estratégia da qualidade. O Seis 
Sigma tem como métodos estatísticos o alicerce para a tomada de decisões, garantindo, assim, uma base científica consistente. A abordagem Seis Sigma muda a forma de gerenciamento, porque as decisões passam a ser abalizadas em dados e, não apenas, em intuições ou sentimentos.

Tabela 1 - Tabela de Conversão Entre ppm e Seis Sigma

\begin{tabular}{c|r|r|r|r}
\hline $\begin{array}{c} \pm \text { Nível sigma do } \\
\text { limite de } \\
\text { especificação }\end{array}$ & $\begin{array}{c}\text { (\%) } \\
\text { Distribuição } \\
\text { Centralizada }\end{array}$ & $\begin{array}{c}\text { *DPMO } \\
\text { Distribuição } \\
\text { Centralizada }\end{array}$ & $\begin{array}{c}\text { (\%) } \\
\text { Distribuição } \\
\mathbf{1 , 5} \text { Sigma }\end{array}$ & $\begin{array}{c}\text { *DPMO } \\
\text { Distribuição } \\
\mathbf{1 , 5} \text { Sigma }\end{array}$ \\
\hline 1 & 68,2689480 & 317310,520 & 30,232785 & 697672,15 \\
\hline 2 & 95,4499876 & 45500,124 & 69,122979 & 308770,21 \\
\hline 3 & 99,7300066 & 2699,934 & 93,318937 & 66810,63 \\
\hline 4 & 99,9936628 & 63,372 & 99,379030 & 6209,70 \\
\hline 5 & 99,9999426 & 0,574 & 99,976733 & 232,67 \\
\hline 6 & 99,9999998 & 0,002 & 99,999660 & 3,4 \\
\hline
\end{tabular}

*DMPO: Defeitos por milhão de oportunidades.

Fonte: Adaptada de BREYFOGLE. (2003, p. 1090-1091).

As ações de um programa Seis Sigma está nos processos, como projetos de produtos e serviços, medidas de desempenho, melhora da eficiência e da satisfação do cliente. Seis Sigma engloba ferramentas e práticas que substituem hábitos reativos por um estilo de gerenciamento dinâmico, receptivo e proativo. Ser proativo significa agir antes dos eventos. No Seis Sigma os métodos utilizados para criar, monitorar e melhorar o sistema de negócios são: (i) gerenciamento do processo; (ii) melhoria do processo; e, (iii) projeto/reprojeto do processo (PANDE, NEUMAN e CAVANAGH, 2001).

Antes de tomar qualquer decisão quanto ao melhoramento do processo, Buss e Ivey (2001), destacam o valor da simulação como ferramenta essencial do Seis Sigma. A simulação permite incorporar testes complexos e regras operacionais para identificar as interações entre os sistemas e os efeitos da variabilidade do processo. Com a utilização da simulação pode-se identificar opções de oportunidade de melhoria, determinar os impactos financeiros e provar a melhor opção de projeto que vise atender todas as expectativas do cliente com um mínimo de capital.

Nesse sentido, a variabilidade é uma medida do grau de dispersão dos resultados dos processos em torno do valor médio. As empresas buscam reduzir continuamente a variabilidade, eliminá-la é impossível. As formas mais comuns de expressar a variabilidade são as medidas estatísticas da amplitude, da variância e do desvio-padrão. O uso de técnicas estatísticas pode ajudar no entendimento da variabilidade e, desta forma, auxiliar as organizações a resolverem os problemas de melhoria na eficácia e eficiência (MARANHÃO, 2001). De acordo com Palmer (1974), o controle e redução da variabilidade dos processos são uma fonte de economia. No entanto, de um modo geral, é mais oneroso obter processos com alta precisão e, então, não se justifica, 
economicamente, trabalhar com tolerâncias que sejam muito grandes em relação à variabilidade do processo.

Para Pande, Neuman e Cavanagh (2001), a variação ajuda a gerência a entender o desempenho real da organização e seus processos. Muitas organizações medem e descrevem seus esforços em termos de médias, mas as médias como, por exemplo, custo médio e tempo de ciclo médio, escondem problemas, ocultando a variação. No Seis Sigma o objetivo é estreitar ou reduzir a variação até que os seis desvios-padrão $(6 \sigma)$ possam ser comprimidos dentro dos limites de especificações do cliente. As medidas Seis Sigma não são estáticas, é necessário ter a consciência de modificar quando as necessidades dos clientes mudam.

Dellaretti Filho e Drumond (1994), enfatizam que a distribuição normal possui a aparência de um gráfico em forma de sino e representa uma distribuição de probabilidade, conforme Figura 2. Essa distribuição é, freqüentemente, adequada para descrever características de qualidade cuja variação é a soma de um grande número de pequenos erros independentes, devido a diferentes causas, tais como os fatores de manufatura. A distribuição normal tem dois parâmetros: (i) centro da distribuição (média), representado pela letra grega $\mu$; e, (ii) dispersão da distribuição (desvio padrão), representada pela letra grega $\sigma$. Cada desvio padrão $(\sigma)$ representa uma área debaixo da curva da distribuição normal, sendo capaz de obter as áreas associadas a cada intervalo como uma proporção da área total sob a curva.

Figura 2 - A Meta dos Seis Sigma

(A) Variação do Processo Igual à Tolerância de Projeto

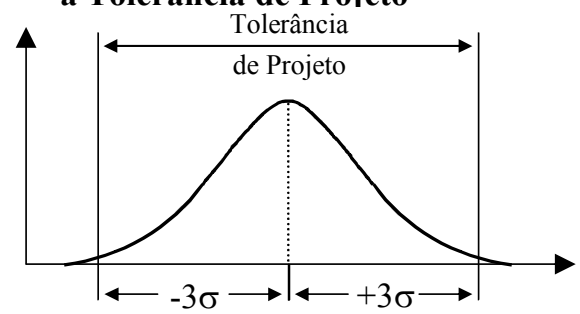

(B) Variação do Processo é Igual

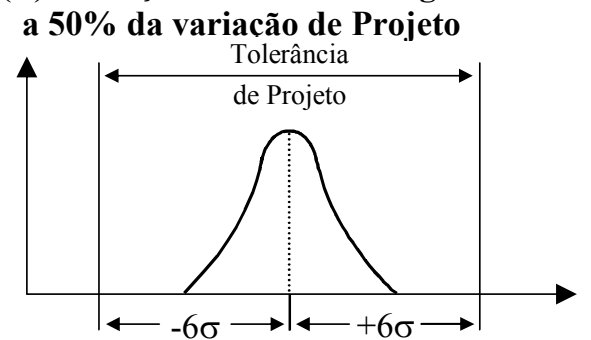

Fonte: DAVIS; AQUILANO; CHASE. (2001, p. 202).

As curvas da distribuição normal ilustram a probabilidade de aceitação de lotes com variação da percentagem de defeituosos. Na Figura 2 estão expostos dois modelos de curvas de distribuição normal. A curva (A) representa o nível $3 \sigma$, com variação do processo igual à tolerância de projeto. Já na curva (B) a variação do processo é igual a $50 \%$ da variação de projeto e representa o nível 6 $\sigma$ (DAVIS, AQUILANO e CHASE, 2001).

Para Davis, Aquilano e Chase (2001), o Controle Estatístico de Processo (CEP) é um método quantitativo para monitorar um processo repetitivo. O CEP coleta dados do processo em 
tempo real e compara as medições atuais com os medidores básicos de desempenho do processo (dados do passado). Através do CEP pode-se analisar a variação do processo e comparar o desempenho atual com o esperado. Desta forma, pode-se estabelecer a Linha Central (LC), ou seja, a média histórica dos dados. Logo após, pode-se definir os Limite Superior de Controle (LSC) e o Limite Inferior de Controle (LIC). O LSC e o LIC são a soma e a diferença, respectivamente, da média histórica e os desvios-padrão. O LSC e LIC são, geralmente, definidos com aproximadamente três desvios-padrão, conforme Figura 3.

Figura 3 - Cálculo dos Limites de Controle

$\begin{aligned} L S C & =\bar{\rho}+3 s \\ L C & =\bar{\rho}=\text { média histórica } \quad s=\sqrt{\frac{\bar{\rho}(1-\bar{\rho})}{n}} \\ L I C & =\bar{\rho}-3 s\end{aligned}$

Fonte: DAVIS; AQUILANO; CHASE. (2001, p. 195).

De acordo com Lourenço Filho (1976), a verificação de que o processo está ou não sob controle é feita pelo exame de amostras extraídas periodicamente. No processo sob controle o característico de qualidade do conjunto dos itens produzidos possui distribuição normal. Quando a variabilidade se torna anormal, as amostras indicarão que o processo de fabricação se modificou e ficou fora de controle. As causas da modificação podem ser descobertas e, por isso, são denominadas causas identificáveis. A presença de causas identificáveis é indicada pela ocorrência de diferenças significantes entre o valor observado e a média do processo, isto é, de valores amostrais fora da faixa de controle. O processo sob controle não possui nenhum ponto fora dos limites, conforme a Figura 4.

Figura 4 - Gráfico de Controle de Qualidade

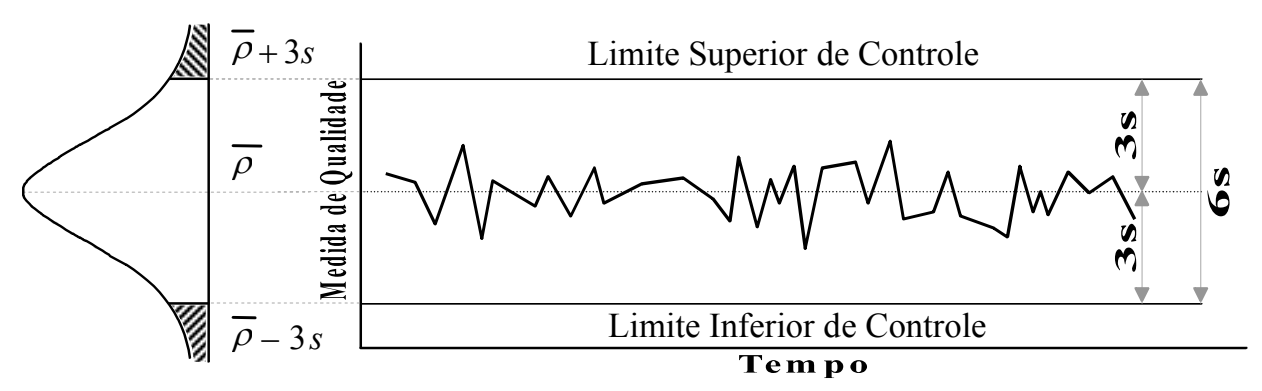

Fonte: Adaptado de SLACK; CHAMBERS; HARLAND; HARRISON; JOHNSTON. (1996, p. 568).

Para Davis, Aquilano e Chase (2001), o objetivo do controle estatístico da qualidade é alcançar um processo tanto sob controle como dentro das tolerâncias. Uma forma rápida de verificar se o objetivo está sendo alcançado é através do uso do coeficiente de capabilidade de processo $\left(C_{p}\right)$. 
A capabilidade é a razão do intervalo de tolerância (LTS - LTI) pela faixa característica do processo $(6 s)$, e pode ser calaculada utilizando a formula exposta na a Figura 5 (A). Slack et al. (1996) destacam que a capabilidade do processo é a medida da aceitabilidade da variação do processo. A capabilidade do processo é dada pela razão entre a faixa de especificação e a variação "natural" do processo, isto é \pm 3 desvios-padrão.

Davis, Aquilano e Chase (2001) ressalvam que o coeficiente de capabilidade de processo $\left(C_{p}\right)$ não indica especificamente quão bem está o desempenho do processo. É preciso calcular o Índice de Capabilidade $\left(C_{p k}\right)$, conforme Figura $5(\mathrm{~B})$, para determinar se a média do processo está próxima ao Limite de Especificação Superior (LES) ou Inferior (LEI). Quando o $C_{p k}$ é igual ao $C_{p}$, então a média do processo está centrada entre os dois limites de especificação. Caso contrário, a média do processo se aproximará ao limite de especificação correspondente ao menor valor resultante do cálculo dos dois coeficientes $C_{p k}$. O índice de capabilidade permite a comparação da faixa característica do processo com as especificações, conforme a Figura 6.

Figura 5 - Coeficiente e Índice de Capabilidade

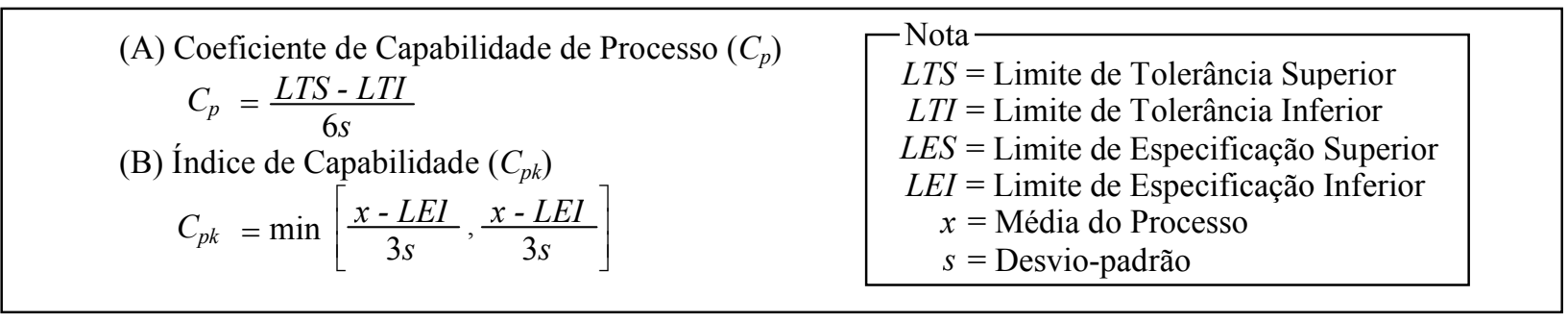

Fonte: DAVIS; AQUILANO; CHASE. (2001, p. 200).

No exemplo da Figura 2, a curva (A) possui tolerância de projeto igual à variação do processo, com um $C_{p}=1,0$. Neste caso, há \pm 3 desvios-padrão de variação de processo dentro da tolerância de projeto. Estes três desvios-padrão representam 99,7\% dentro das especificações ou 2.700 defeitos por milhão. Já na curva (B) da Figura 2, que representa o programa Seis Sigma, a variação do processo é reduzida a $50 \%$ da tolerância de projeto. Isto resulta em um $C_{p}=2,0$ e somente 3,4 ppm.

Figura 6 - Tolerância e Especificação

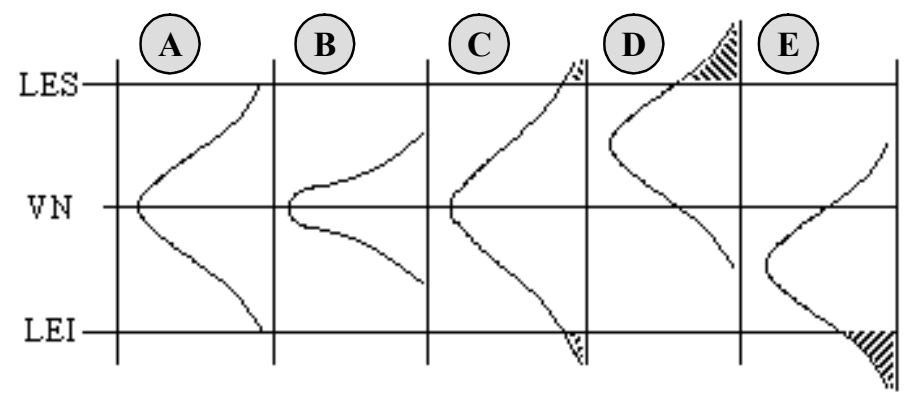

Fonte: Adaptado de LOURENÇO FILHO. (1976, p. 66). 
Existe uma distinção entre os Limites de Especificação ou Tolerância (LES, VN, LEI) e os Limites de Controle (LSC, LC, LIC). Segundo Lourenço Filho (1976), os limites da especificação representam aquilo que se exige no projeto, para que o produto possa atender à finalidade para a qual é desejado. Os limites de controle resultam do processo de fabricação empregado e refletem aquilo que o processo é capaz de realizar. Portanto, de posse dos limites de controle e de especificação, já é possível verificar se o processo pode ou não atender à especificação. Na Figura 6, os casos (A), (B) e (C) identificam que a média do processo de fabricação coincide com o valor nominal da especificação. Essa coincidência significa que o nível médio de qualidade é satisfatório, muito embora a dispersão possa ser maior do que a especificação. Quando a média do processo estiver acima do valor nominal (D) ou abaixo dele (E), então, será indicativo de elevada porcentagem de peças inaceitáveis (área hachurada). Quando o processo não atende à tolerância da especificação, pode-se tomar as seguintes decisões: (i) modificar a especificação; (ii) alterar o processo de fabricação; e, (iii) empregar inspeção completa, com triagem das peças fora da especificação.

Uma distribuição normal com $C_{p}>1\left(C_{p}=\right.$ coeficiente de capabilidade de processo $)$ é considerada indicativa de que o processo é "capaz" e quando a $C_{p}<1$, indica que o processo é "nãocapaz", conforme demonstrado nos gráficos (A) e (C) expostos na Figura 7. A simples medida de $C_{p}$ pressupõe que a média da variação do processo está no ponto médio da faixa de especificação. Porém, com freqüência a média do processo é viesada em relação à faixa de especificação, ou seja, tende ao Limite de Tolerância Superior (LTS) ou para o Limite de Tolerância Inferior (LTI), conforme Figura 7 (D). Nesses casos, é necessário calcular os índices de capabilidade $C_{p k}$ para compreender a capabilidade do processo $C_{p}$ (SLACK et al., 1996).

Figura 7 - Capabilidade do Processo
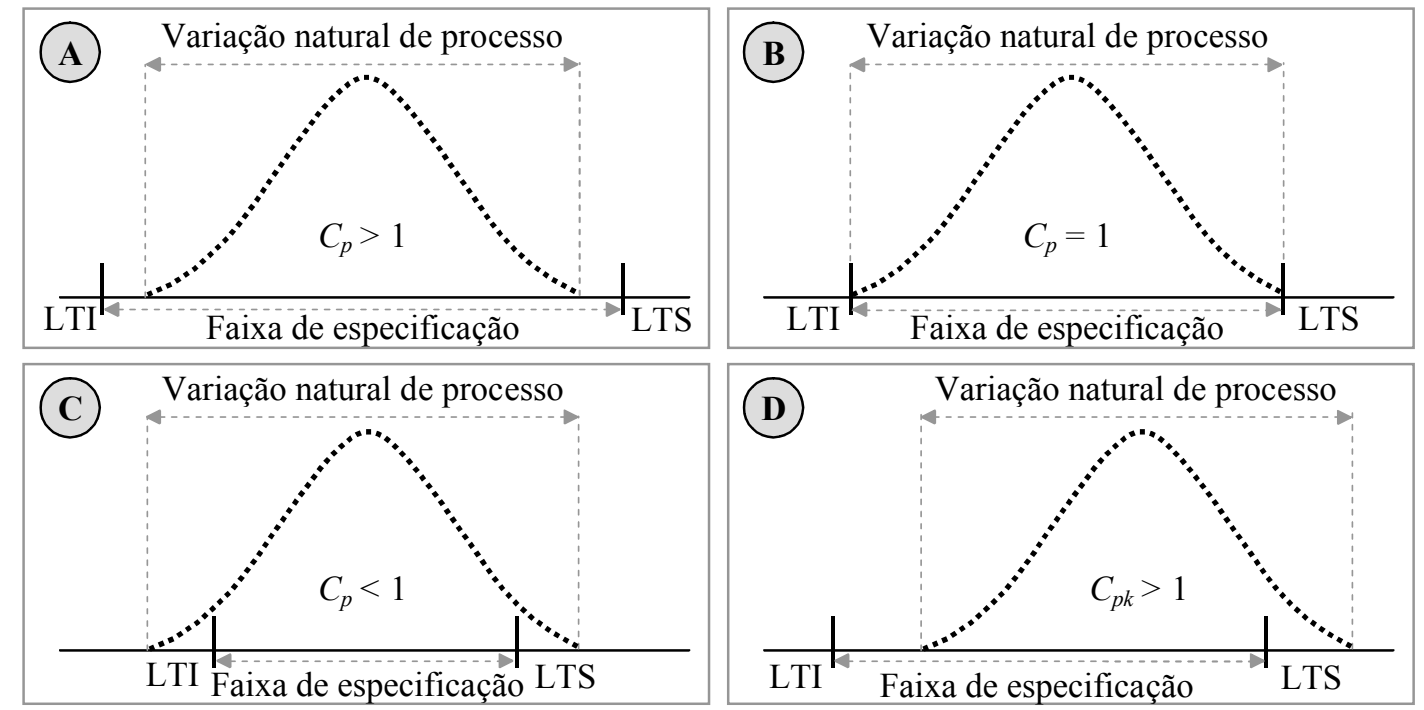

Fonte: Adaptado de SLACK; CHAMBERS; HARLAND; HARRISON; JOHNSTON. (1996, p. 564). 
O processo pode estar sob controle estatístico e não ser capaz, ou seja, na média, poderá estar produzindo itens dentro dos limites de controle, mas sua variação é tanta que não consegue atender as especificações para todos os itens, conforme Figura 8 - (A). No entanto, o processo com variância reduzida não garante uma produção livre de defeitos. Com a redução da variabilidade, os limites de controle para as médias da amostra estão contidos nos limites de tolerância, ou seja, diminui os itens fora dos limites de tolerância, conforme figura $8-(\mathrm{B})$. Quando a variabilidade do processo é conduzida ao controle, o processo é capaz de realizar produção livre de defeitos. Neste caso, a variância é reduzida a tal ponto que nenhuma medida individual poderá cair fora da tolerância, conforme Figura 8 - (C). Para conseguir reduzir a variância do processo até que todos os componentes estejam dentro das especificações (livre de defeitos) é necessário melhorar o desempenho de cada fonte de variação. Estas fontes de variação são: operadores, máquinas, ferramentas, setup, material e o meio-ambiente (DAVIS, AQUILANO e CHASE, 2001).

Figura 8 - Redução da Variância do Processo

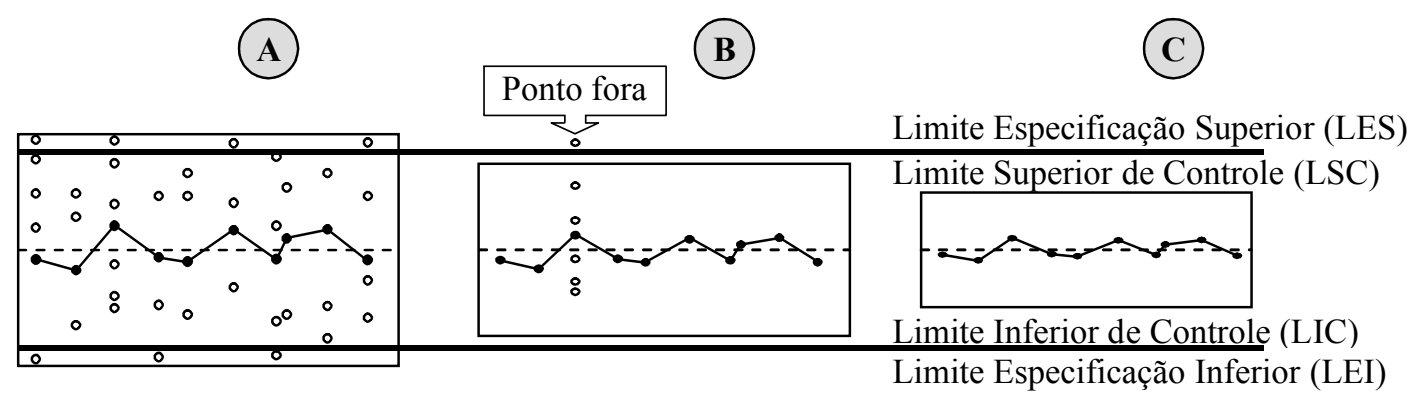

Fonte: DAVIS; AQUILANO; CHASE. (2001, p. 200).

O fluxo de processo das empresas Seis Sigma é composto pelas entradas do processo, o processo em si, representado por um mapa de fluxograma, e as saídas, representadas por clientes, produtos finais e lucros. A Figura 9 ilustra essa relação, as letras (x), que aparecem na entrada e no fluxograma do processo seriam indicadores de mudanças ou desempenho nas partes independentes do sistema. Já os (y) representam medidas do desempenho dos negócios. A fórmula $\mathrm{y}=\mathrm{f}(\mathrm{x})$ é uma maneira matemática de representar que mudanças nas variáveis de entrada e processos do sistema (x) determinarão os resultados dos negócios (y), ou seja, os resultados dos negócios estão em função das entradas e do fluxo do processo. Portanto, é necessário compreender qual as variáveis (x) da entrada e do processo de negócios têm maior influência sobre os (y) ou resultados. Desta forma pode-se usar as mudanças no desempenho geral do processo para ajustar os negócios e mantê-los em movimento sobre um caminho lucrativo (PANDE, NEUMAN e CAVANAGH, 2001). 
Pande, Neuman e Cavanagh (2001) afirmam que é essencial um procedimento para sustentar a melhoria Seis Sigma. Esse procedimento ou caminho para a melhoria Seis Sigma pode ser definido em 5 (cinco) etapas: (i) identificar processos essenciais e clientes-chave; (ii) definir necessidades de clientes; (iii) medir desempenho atual; (iv) priorizar, analisar e implementar melhorias; e, (v) expandir e integrar o sistema Seis Sigma.

Figura 9 - Variáveis Independentes (x) e Dependentes (y)

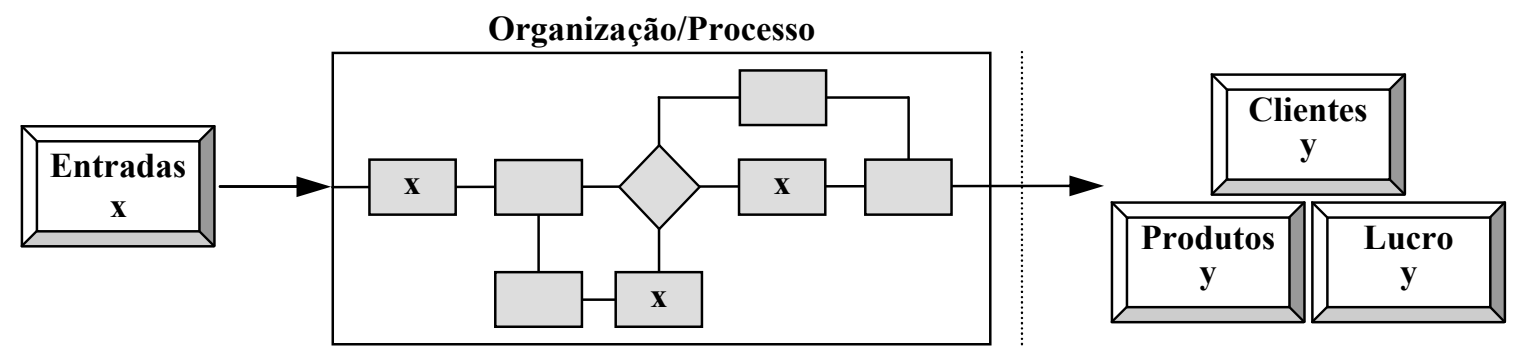

Fonte: PANDE; NEUMAN; CAVANAGH. (2001, p. 23).

Uma das ferramentas utilizadas na gestão da qualidade é o PDCA. Campos (1992) define o PDCA como sendo um método para a prática de controle, ou seja, o controle de processo é exercido através do ciclo PDCA. Os termos desse ciclo têm os seguintes significados: $(\mathrm{P}=$ Plan $)$ planejamento - consiste em estabelecer metas sobre os itens de controle e as diretrizes para se atingir as metas; $(\mathrm{D}=\mathrm{Do})$ Execução - efetivar as tarefas e coletas de dados para verificação; $(\mathrm{C}=$ Check) verificação - comparar os resultados com as metas planejadas; $(\mathrm{A}=$ Action) atuação corretiva - detectar e definir as ações corretivas de tal forma que o problema seja extinto. Segundo Pande, Neuman e Cavanagh (2001), o modelo de melhoria do Seis Sigma baseia-se no PDCA. Na estratégia Seis Sigma o ciclo de melhoria compreende as seguintes fases: Definir, Medir, Analisar, Melhorar e Controlar, ou simplesmente DMAIC. Para Breyfogle (2003) as cinco fases do ciclo de melhoria baseado no Seis Sigma são: $(\mathrm{D}=$ Define Project) definição do projeto; $(\mathrm{M}=$ Measure) mensurar e avaliar o projeto; (A = Passive Analysis), analise passiva; ( $=$ Proactive Testing) desenvolver ensaios, métodos e processos de ação pró-ativa; e, $(\mathrm{C}=$ Control $)$ controlar os resultados.

Rivera e Marovich (2001) afirmam que a metodologia DMAIC representa as fases fundamentais no desenvolvimento de projeto Seis Sigma. Essas fases garantem que as empresas apliquem a técnica em um metódico e disciplinado caminho, bem como a correta definição e execução dos projetos e incorporação dos resultados alcançados com eles. Os autores afirmam que a fase de definição é crítica e fundamental na garantia de sucesso do projeto Seis Sigma.

Ao analisar, no entanto, as "Organizações Seis Sigma”, percebe-se que não existem empresas que atingiram a qualidade no nível Seis Sigma em todas as áreas e departamentos. Nenhuma empresa possui mais do que alguns processos neste nível. Usar medidas sigma ou alguma 
outra ferramenta não qualifica uma empresa como sendo uma "Organização Seis Sigma". Para tornar-se uma organização Seis Sigma é necessário que a companhia assuma o desafio de medir e melhorar todos os processos. Todas as empresas querem resultados do Seis Sigma, mas o tipo de resultado ou mudança pode variar muito. As empresas implementam o sistema Seis Sigma para buscar resultados baseados em diversos objetivos: (i) transformação do negócio (mudança de cultura, mudança estrutural, atitudes focalizadas no cliente); (ii) melhoria estratégica (acelerar o desenvolvimento de produtos, eficiência da cadeia de suprimento); e, (iii) solução de problemas (altos custos, retrabalhos, atrasos). A busca por resultados satisfatórios em um sistema Seis Sigma está estreitamente ligado à seleção de projetos. Projetos de melhoria bem selecionados e bem definidos são iguais a resultados melhores (PANDE, NEUMAN e CAVANAGH, 2001).

Segundo Berdebes (2003), o sucesso do programa Seis Sigma depende de profissionais devidamente treinados, que possuem o domínio de várias ferramentas de qualidade e de avançadas análises estatísticas. Estes profissionais são denominados Master Black Belts, Black Belts e Green Belts ou, respectivamente, mestre faixa-preta, faixa-preta, faixa-verde. Os Master Black Belts estão aptos a treinar os Black Belts e Green Belts nos métodos estatísticos analíticos e são especialistas na metodologia Seis Sigma. Os Black Belts estão ativamente envolvidos na organização, são considerados os comandantes técnicos, uma de suas tarefas é a extração de dados para análise. Os Green Belts são responsáveis pela administração de todas as facetas dos projetos Seis Sigma como, por exemplo, gestão de reuniões, gerenciamento de projetos, técnicas de controle de qualidade e análise de dados elementares.

Harry e Schroeder (2000) afirmam que os títulos Master Black Belts, Black Belts, e Green Belts foram criados na Motorola e procuram distinguir as qualificações desses profissionais que administram a qualidade. Existem outras empresas que adotaram os títulos Yellow Belts e White Belts aos termos já citados, isto se deve a forte tendência das organizações em personalizar e criar mais níveis de profissionais em seus programas de Seis Sigmas. McCarthy e Stauffer (2001), afirmam que, tradicionalmente, os Black Belts utilizam muito a estatística nos seus projetos. Recentemente, os Black Belts introduziram a simulação para o projeto seis sigma, e a solução dessa simulação é considerada parte do "kit de ferramentas Seis Sigma".

\section{Aspectos Metodológicos}

No desenvolvimento deste artigo foi utilizada uma pesquisa exploratória a fim de investigar os benefícios do programa Seis Sigma em relação a duas etapas do processo de fabricação do refrigerante pet 2 litros. Segundo Gil (2002), este método de pesquisa é utilizado para investigar e proporcionar uma visão geral, de tipo aproximativo, acerca do problema proposto. Foram usados 
para este fim: (i) dados primários extraídos junto a uma fábrica de refrigerantes franqueada a uma multinacional; e, (ii) dados secundários extraídos da internet, pesquisa bibliográfica e pesquisa documental. O método utilizado para calcular o nível sigma e o percentual de rendimento do processo é o mesmo adotado por Pande, Neuman e Cavanagh (2001) e Breyfogle (2003).

Os dados primários são compostos por 610 amostras de refrigerantes pet $2000 \mathrm{ml}$ que, por sua vez, foram submetidas a testes de 10 itens de controle. Esses itens de controle são, normalmente, verificados e analisados estatisticamente pelo pessoal responsável pelo CEP Controle Estatístico do Processo da empresa, a fim de verificar se o processo está sob controle e dentro das especificações. Os itens de controle podem ser divididos em dois sub-processos, um referente ao envase e, o outro referente à injeção plástica da garrafa pet (sopro). Para o sub-processo de envase foram coletados os dados de volume líquido de refrigerante; para o sub-processo de injeção plástica da garrafa pet foram coletados os seguintes dados: (i) ponto de injeção; (ii) área de fundo; (iii) petalóide; (iv) calcanhar; (v) cintura; (vi) parede lateral inferior; (vii) painel do rótulo; (viii) ombro; e, (ix) ombro superior. Outras informações fornecidas pelo departamento de qualidade da empresa dizem respeito às especificações desses itens de controle relacionados anteriormente.

\section{Estudo de Caso: O Processo de Fabricação do Refrigerante Pet 2000 ml}

Como este estudo possui, aproximadamente, 6.000 medidas de verificação (10 itens de controle, cada um com 610 medidas de verificação) fica difícil expor todos esses dados e seus respectivos cálculos, sendo impossível apresentar todos as informações neste instrumento de pesquisa. Entretanto, será exposta a metodologia dos cálculos utilizados, de forma simplificada e genérica, para que se possa evidenciar como foram estimados os resultados desta pesquisa.

Foram criados gráficos de controle para todos os itens de controle deste estudo, bem como plotados as especificações dos mesmos. Logo após, foi construído uma matriz que possibilitasse a identificação dos pontos que estavam fora das especificações para cada item de controle, desta forma foi possível identificar a quantidade de pontos fora dos limites de especificações (oportunidades) de cada amostra, conforme o modelo da Figura 10.

Figura 10 - Modelo de matriz de verificação de oportunidades de defeitos

\begin{tabular}{|c|c|c|c|c|c|c|c|c|c|c|c|}
\hline Amostra & A & B & C & D & E & F & G & H & I & J & Oportunidade \\
\hline $\mathbf{1}$ & & & $\mathrm{X}$ & $\mathrm{X}$ & & & & & & & 2 \\
\hline $\mathbf{2}$ & & & & & & & & & & & 0 \\
\hline $\mathbf{3}$ & & & & $\mathrm{X}$ & & & $\mathrm{X}$ & & & & 2 \\
\hline $\mathbf{4}$ & & & & & & & & & & & 0 \\
\hline $\mathbf{5}$ & $\mathrm{X}$ & & $\mathrm{X}$ & $\mathrm{X}$ & & $\mathrm{X}$ & & $\mathrm{X}$ & & & 5 \\
\hline Total & 1 & 0 & 2 & 3 & 0 & 1 & 1 & 1 & 0 & 0 & 9 \\
\hline
\end{tabular}

Fonte: Desenvolvido pelos autores. 
No caso das 610 amostras a matriz foi construída com o auxílio de planilha eletrônica para facilitar o cálculo do número de oportunidades para cada amostra e para cada item de controle. Foram, também, estimados os percentuais de rendimento para cada item de controle, bem como seus respectivos níveis sigma e cálculo de defeitos por milhão de oportunidades (DPMO), conforme a Figura 11.

A identificação de todas as oportunidades de defeitos de cada item de controle e do total dos defeitos das 610 amostras, conforme Figura 11, serviram como subsídios para calcular o rendimento percentual do processo como um todo e seu respectivo nível sigma. Para determinar a estimativa do rendimento percentual e do nível sigma do processo foi utilizado o mesmo método de cálculo aplicado nos sub-processos (10 itens de controle), conforme Figura 12.

Figura 11 - Matriz de verificação de oportunidades de defeitos do processo pet $2000 \mathrm{ml}$

\begin{tabular}{|c|c|c|c|c|c|c|c|c|c|c|c|}
\hline Amostra $\downarrow$ & $\mathbf{A}$ & $\mathbf{B}$ & $\mathbf{C}$ & $\mathbf{D}$ & $\mathbf{E}$ & $\mathbf{F}$ & $\mathbf{G}$ & $\mathbf{H}$ & $\mathbf{I}$ & J & Oportunidade \\
\hline $\mathbf{0 0 1}$ & & & $\mathrm{X}$ & & & & & & & & 1 \\
\hline $\mathbf{0 0 2}$ & & & & & & & & & & & 0 \\
\hline $\mathbf{0 0 3}$ & & & $\mathrm{X}$ & $\mathrm{X}$ & $\mathrm{X}$ & $\mathrm{X}$ & $\mathrm{X}$ & $\mathrm{X}$ & $\mathrm{X}$ & $\mathrm{X}$ & 8 \\
\hline $\mathbf{0 0 4}$ & $\mathrm{X}$ & & & $\mathrm{X}$ & & & $\mathrm{X}$ & $\mathrm{X}$ & & & 4 \\
\hline $\mathbf{0 0 5}$ & & & & & & & & & & & 0 \\
\hline $\mathbf{. .}$ & & & & & & & & & & & 0 \\
\hline $\mathbf{6 1 0}$ & & & & & $\mathrm{X}$ & & & & $\mathrm{X}$ & & 2 \\
\hline Total & 4 & 0 & 73 & 60 & 116 & 31 & 26 & 30 & 15 & 9 & 364 \\
\hline DPMO & 6557 & 0 & 11967 & 98361 & 19016 & 50820 & 42623 & 49180 & 24590 & 14754 & \multirow{2}{*}{} \\
\hline Sigma & 3 & 6 & 2,7 & 2,8 & 2,4 & 3,1 & 3,2 & 3,2 & 3,5 & 3,7 & \\
\hline R (\%) & 99,34 & 100,0 & 88,03 & 90,16 & 80,98 & 94,92 & 95,74 & 95,08 & 97,54 & 98,52 & \\
\hline
\end{tabular}

Nota: DMPO = Defeitos por milhão de oportunidades; R (\%) = Rendimento (\%); A = volume de envase de refrigerante; $\mathrm{B}=$ ponto de injeção; $\mathrm{C}=$ área de fundo; $\mathrm{D}=$ petalóide; $\mathrm{E}=$ calcanhar; $\mathrm{F}=$ cintura; $\mathrm{G}=$ parede lateral inferior; $\mathrm{H}=$ painel do rótulo; $\mathrm{I}=$ ombro; $\mathrm{e}, \mathrm{J}=$ ombro superior.

Fonte: Desenvolvido pelos autores.

Deve ficar bem claro que o total de defeitos (364) encontrados nas 610 amostras são referentes aos 10 itens de controles submetidos a esse estudo, ou seja, não pode-se afirmar que existem 364 amostras com defeitos em 610 analisadas, mas sim que há 364 defeitos nas 610 amostras. Portanto, são coisas totalmente diferentes e que sua distinção deve ficar bem nítida para que haja um claro entendimento do método de determinação da estimativa do nível sigma do processo.

Figura 12 - Rendimento Percentual e Nível Sigma do Processo

(A) Oportunidades Totais $(O T)$

$$
O T=U C x O
$$

(B) Defeitos Por Milhão de Oportunidades (DPMO)

$$
D P M O=(D T C / O T) \times 10^{6}
$$

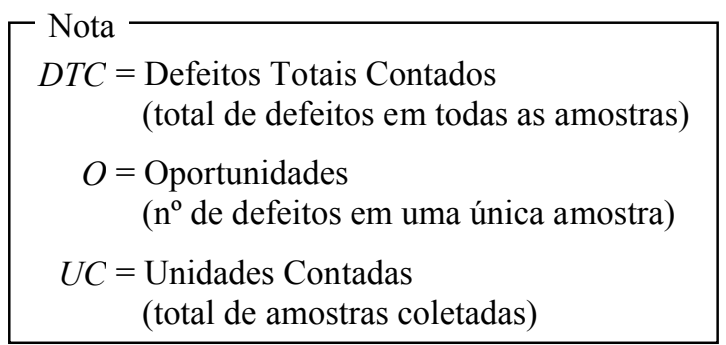


As informações obtidas através da construção da matriz de verificação de oportunidades (Figura 11) foram utilizadas para calcular e determinar o rendimento percentual e o nível sigma do total do processo em estudo (todos os 10 itens de controle), conforme Tabela 2. O nível sigma é determinado através de uma tabela de conversão do rendimento percentual do processo, de acordo com o modelo de Breyfogle (2003) (Tabela 1). É importante lembrar que este método de cálculo do sigma é uma estimativa e, portanto, o número sigma pode variar significativamente com base na precisão de seus dados e no número de oportunidades identificadas em uma unidade (amostra).

Tabela 2 - Informações do Processo de Fabricação do Refrigerante Pet $2000 \mathrm{ml}$

\begin{tabular}{l|r}
\hline \multicolumn{1}{c|}{$\begin{array}{c}\text { Informações dos 10 Itens de Controle do Processo de } \\
\text { Fabricação do Refrigerante Pet 2000 } \mathbf{~ m l}\end{array}$} & \multicolumn{1}{c}{ Dados } \\
\hline Total de dados coletados - UC & 610 \\
\hline Total de defeitos - DTC & 364 \\
\hline Oportunidades - O & 8 \\
\hline Oportunidades totais - OT & 4880 \\
\hline Defeitos por milhão de oportunidades - DPMO & $74.590,16$ \\
\hline Nível sigma (aproximado) & 2,95 \\
\hline Rendimento do processo (\%) & 92,54098 \\
\hline
\end{tabular}

Fonte: Desenvolvido pelos autores.

Através dos dados da Tabela 2 pode-se considerar que o processo em estudo está, aproximadamente, 92,54\% em conformidade com as especificações e que a conversão para o nível sigma evidenciou um processo 2,95 $\sigma$. A empresa em estudo produz 3.428 .100 unidades/mês de pet $2000 \mathrm{ml}$, um total de 41.137.200 unidades/ano. Com o rendimento de 92,54098\% a empresa produz, aproximadamente, 38.068.768 refrigerantes pet $2000 \mathrm{ml}$ com nenhum tipo de defeito ou 3.068.432 refrigerantes com pelo menos um defeito identificável. A mesma produção de 41.137.200 unidades/ano de refrigerantes pet $2000 \mathrm{ml}$ no nível de qualidade Seis Sigma teria uma estimativa de rendimento próximo de $100 \%(99,99966 \%)$ ou um total de 140 unidades de refrigerantes com defeitos em todo o ano de fabricação, conforme evidenciado na Tabela 3.

Tabela 3 - Comparação do Nível Sigma do Processo

\begin{tabular}{l|r|r}
\hline Dados comparativos & Processo atual & \multicolumn{1}{c}{ Processo estimado } \\
\hline Nível Sigma & 2,95 & 6,00 \\
\hline Rendimento (\%) & 92,54098 & 99,99966 \\
\hline Total produzido por ano & 41.137 .200 & 41.137 .200 \\
\hline Produtos sem nenhum defeito (unid./ano) & 38.068 .768 & 41.137 .060 \\
\hline Produtos com pelo menos um defeito (unid./ano) & 3.068 .432 & 140 \\
\hline Resultado de melhoria no processo & $\mathbf{3 . 0 6 8 . 2 9 2}$ unidades/ano \\
\hline
\end{tabular}


Fonte: Desenvolvido pelos autores.

\section{Considerações Finais}

Criar estratégias que tragam benefícios para as organizações requer do administrador uma habilidade prática e teórica bastante relevante, visto que existem várias estratégias e ferramentas que estão sendo empregadas nos mais diversos meios organizacionais. O mais difícil não é identificar as estratégias que podem ser adotadas pela organização, mas sim evidenciar as vantagens e desvantagens intrínsecas à estratégia e mensurar os benefícios que ela pode proporcionar. O Seis Sigma é mais uma dessas estratégias ou ferramentas de gestão que vem sendo empregada de forma bastante participativa. Existem vários estudos que demonstram os benefícios que as organizações alcançam com a implantação da estratégia Seis Sigma, mas há, também, relatos de fracassos. Grande parte desse fracasso está atrelada ao alto custo de implementação do programa Seis Sigma, pois o investimento em treinamento não é muito barato e, muitas vezes, o retorno não aparece. A chave para o sucesso de um programa Seis Sigma está na escolha de projetos que possibilitem retornos à altura do investimento.

No caso deste estudo, o processo de fabricação do refrigerante pet 2 litros é composto por vários sub-processos, e não somente o envase e a injeção plástica da garrafa, pois existem outras atividades que compõem o produto final e que deveriam fazer parte do estudo de melhoria do processo. Seria mais relevante se fosse considerado outras informações, desde a entrada dos insumos, passando por todo o processo de fabricação, até chegar ao cliente final. Portanto, pode-se afirmar que o envase e a injeção plástica da garrafa pet 2 litros é dois sub-processos, não sendo ideal para formar um projeto Seis Sigma. É preciso avaliar todo o processo, desta forma, pode-se obter uma noção mais realista dos benefícios que a empresa poderia alcançar.

Em se tratando apenas de duas etapas do processo de fabricação do refrigerante pet 2 litros, e assumindo a premissa de que a empresa alcance o nível Seis Sigma, a economia operacional pode chegar a 3.068.292 unidades pet por ano, ou seja, aproximadamente, 255.691 refrigerantes por mês estarão dentro dos limites de especificação. Há, também, um benefício implícito que o programa Seis Sigma pode proporcionar, através da diminuição da variabilidade do volume de refrigerante envasado, a empresa consegue comprimir a faixa característica do processo, reduzindo, assim, os limites de especificação. Desta forma, a economia poderia chegar, também, na quantidade de refrigerante envasado. Esse mesmo raciocínio poderia ser admitido para a quantidade de plástico injetado, com a diminuição da variabilidade e, conseqüentemente, a redução de matéria-prima plástica.

A grande questão está na análise do investimento necessário para implantar a filosofia Seis Sigma, pois em se tratando apenas no objeto deste estudo, os benefícios operacionais são relevantes, 
basta, agora, transformar os ganhos operacionais em ganhos financeiros e realizar a confrontação desses ganhos com o investimento total necessário para sua implementação. Neste estudo, ficou claro que em termos operacionais o programa Seis Sigma pode trazer grandes benefícios.

\begin{abstract}
In this context for searching of perfect products, there are many strategies that aim and improve the competitive advantages of organizations, that includes the Six Sigma. This article aims to compare the operationals benefits which Six Sigma program can proportionate in the present production process of pet refrigerant $2000 \mathrm{ml}$ of X company. In development of this article was utilized an explorer method in order to investigate and proportionate a general vision as kind of approach relationed with the Six Sigma program in relation to the manufacture process of pet $2000 \mathrm{ml}$ refrigerant. In present study, was evident that in operationals conditions, the Six Sigma program can bring if as good applicable is great benefits to this production process of pet 2 liters refrigerant, reducing then, number of fails and, consequently, increasing the uniformity of products.
\end{abstract}

Key-words: Six Sigma; Quality Control; Management Process.

\title{
Referências
}

BATEMAN, T. S.; SNELL, S. A. Administração: construindo vantagem competitiva. São Paulo: Atlas, 1998.

BERDEBES, G. Introduction to six sigma: for internal service departments and professional services organizations. Quebec: Working Paper, 2003.

BOECHAT, Y. Faixas-pretas economizam bilhões nas empresas. Jornal Gazeta Mercantil: empresas e carreiras, São Paulo, 21 mar. 2000.

BREYFOGLE, F. W. Six Sigma: smarter solutions using statistical methods. 2. ed. Austin: Wiley, 2003.

BUSS, P.; IVEY, N. Dow chemical design for six sigma rail delivery project. In: Proceeding of the 2001 Winter Simulation Conference, Phoenix, Arizona, 2001.

CAMPOS, M. S. Em busca do padrão seis sigma. Revista Exame, São Paulo, edição 689, ano 32, n.11, 02 jun. 1999.

CAMPOS, M. S. Seis sigma: presente e futuro. In: Congresso SAE Brasil, São Paulo, 2002.

CAMPOS, V. F. Controle da qualidade total: no estilo japonês. Belo Horizonte: Fundação Christiano Ottoni, 1992.

DAVIS, M. M.; AQUILANO N. J.; CHASE R. B. Fundamentos da administração da produção. 3. ed. Porto Alegre: Bookman, 2001.

DELlARETTI FILHO, O. D.; DRUMOND, F. B. Itens de controle e avaliação de processos. Belo Horizonte: Fundação Christiano Ottoni, 1994.

GARVIN, D. A. Gerenciando a qualidade: a visão estratégica e competitividade. Rio de Janeiro: Qualitymark, 1992.

GIL. A. C. Como elaborar projetos de pesquisa. 4. ed. São Paulo: Atlas, 2002.

HARRY, M.; SCHROEDER, R. Six sigma: the breakthrough management strategy revolutionizing the world's top corporations. Nova York: Doubleday, 2000.

HSM MANAGEMENT. Dossiê 6-sigma: a um passo da perfeição. Revista HSM Management. São Paulo: HSM do Brasil, ano 7, n. 38, mai./jun. 2003. p. 63-90.

HUTCHINS, G. ISO 9000: um guia completo para o registro, as diretrizes da auditoria e a certificação bem-sucedida. São Paulo: Makron Books, 1994. 
LOBOS, J. Qualidade através das pessoas. 9. ed. São Paulo: Instituto da Qualidade, 1991.

LOURENÇO FILHO, R. C. B. Controle estatístico de qualidade. Rio de Janeiro: Livros Técnicos e Científicos, 1976.

MARANHÃO, M. ISO série 9000: manual de implementação - versão 2000. 6. ed. Rio de Janeiro: Qualitymark, 2001.

McCARTHY, B. M.; STAUFFER, R. Enhancing six sigma through simulation with igrafx process for six sigma. In: Proceeding of the 2001 Winter Simulation Conference, Phoenix, Arizona, 2001.

PALMER, C. F. Controle total de qualidade. São Paulo: Editora da Universidade de São Paulo, 1974.

PANDE, P. S.; NEUMAN, R. P.; CAVANAGH, R. R. Estratégia seis sigma: como a GE, a Motorola e outras grandes empresas estão aguçando seu desempenho. Rio de Janeiro: Qualitymark, 2001.

RIVERA, A.; MAROVICH, J. Use of six sigma to optimize cordis sales administration and order and revenue management process. In: Proceeding of the 2001 Winter Simulation Conference, Phoenix, Arizona, 2001.

SLACK, N.; CHAMBERS, S.; HARLAND, C.; HARRISON, A.; JOHNSTON, R. Administração da Produção. São Paulo: Atlas, 1996.

\section{Dados do primeiro autor:}

Nome: Sidarta Ruthes

Filiação institucional: Universidade Tecnológica Federal do Paraná - UTFPR

Departamento: Programa de Pós-Graduação em Tecnologia - PPGTE

Função: Mestrando em Tecnologia

Endereço: Av. Cândido de Abreu, 469 - Cj. 1305 - Centro Cívico - Curitiba - Paraná - Brasil CEP: 80530-000

Telefones: (41) 8413-3830

e-mail:sidarta@ppgte.cefetpr.br

Recebido para publicação: 30/03/2006

Aceito para publicação: 05/06/2006 